\title{
КАК ИЗМЕРИТЬ КАЧЕСТВО ЭНДОСКОПИЧЕСКОЙ РЕТРОГРАДНОЙ ХОЛАНГИОПАНКРЕАТОГРАФИИ (ЭРХПГ)
}

\author{
Аннаоразов Ылхам Аиирович \\ Международный казасхко-туреикий университет имени Х.А. Ясави, \\ г.Шымкент, Казахстан \\ Жакипов Нуркен Серикович \\ Областная клиническая больница Туркестанской области, \\ г.Шымкент, Казахстан \\ Есалиева Рахила Имашовна \\ Областная клиническая больница Туркестанской области, \\ Аннаоразова Зарина Ихтиаркизи \\ г.Шымкент, Казахстан \\ Международный казасхко-турецкий университет имени Х.А. Ясави, \\ г.Шымкент, Казахстан \\ Annarazov Ylham Ashirovich \\ H.A. Yasavi International Kazakh-Turkish University, \\ Shymkent, Kazakhstan \\ Zhakipov Nurken Serikovich \\ Regional Clinical Hospital of Turkestan region, \\ Shymkent, Kazakhstan \\ Yesalieva Rakhila Imashovna \\ Regional Clinical Hospital of Turkestan region, Shymkent, Kazakhstan \\ Annaorazova Zarina Ihtiarkizi \\ H.A. Yasavi International Kazakh-Turkish University, \\ Shymkent, Kazakhstan
}

\begin{abstract}
Аннотация. Эндоскопическая ретроградная холангиопанкреатография (ЭРХПГ) является малоинвазивной терапевтической процедурой, требующей особого индивидуального подхода к обучению и освоению. Это связано с большей заболеваемостью и смертностью, чем любая другая процедура в гастроэнтерологии. Риск осложнений и вреда для пациента неизбежно требует соблюдения строгих рекомендаций в отношении обучения, сертификации и квалификации. В попытке снизить риск осложнений различные эндоскопические общества по всему миру разработали так называемые “Меры качества”, которые оказались основой безопасности и качества ЭРХПГ.

Abstract. Endoscopic retrograde cholangiopancreatography (ERCP) is a minimally invasive therapeutic procedure that requires a special individual approach to learning and mastering. This is associated with greater morbidity and mortality than any other procedure in gastroenterology. The risk of complications and harm to the patient inevitably requires compliance with strict guidelines regarding training, certification and qualifications. In an attempt to reduce the risk of complications, various endoscopic societies around the world have developed so-called "Quality Measures", which have proved to be the basis for the safety and quality of ERCP.
\end{abstract}

Ключевые слова: Эндоскопическая ретроградная холангиопанкреатография (ЭРХПГ); качество; осложнения

Keywords: Endoscopic retrograde cholangiopancreatography (ERCP); quality; complications

Вступление

Эндоскопическая ретроградная холангиопанкреатография (ЭРХПГ) - это эндоскопическая методика, разработанная в 1960-х годах для диагностики патологии поджелудочной железы. С момента своего появления она превратилась из чисто диагностической в преимущественно терапевтическую процедуру. Она зарекомендовала себя как одна из самых сложных и технически сложных процедур в эндоскопии желудочнокишечного тракта, требующая специальной подготовки и обучения для освоения. В последние годы было опубликовано множество руководств по измерению качества ЭРХПГ. Эра Интернета сделала его широко доступным для пациентов, чтобы исследовать и спрашивать об уровне успеха их оператора в выполнении процедуры и частоте осложнений. До недавнего времени частота осложнений была единственным показателем для оценки результатов, поскольку ЭРХПГ связана с большей заболеваемостью и смертностью по сравнению с другими эндоскопическими процедурами, такими как колоноскопия и гастроскопия. Вскоре стало очевидно, что необходимо разработать дополнительные меры для оценки качества ЭРХПГ. 
В связи с необходимостью дополнительных мер Американское общество эндоскопии желудочнокишечного тракта (ASGE) провело консенсусную конференцию в 2001 году в попытке определить меры, которые будут полезны для отслеживания результатов работы эндоскопистов в повседневной практике. Они разделили меры на три группы: структура, процесс и результаты. Там, где структура представляет характеристики пациента-эндоскописта перед процедурой, процесс представляет технический аспект ЭРХПГ, а результат оценивает пациента после процедуры. Эта конференция послужила ориентиром для всех последующих разработанных руководящих принципов.

Наиболее известные руководящие принципы ASGE и Европейского общества эндоскопии желудочнокишечного тракта (ESGE) по показателям качества имеют в качестве основы те же меры, разделенные на предпроцедурные, внутрипроцедурные и послепроцедурные [1,2].

\section{Предпроцедурный период}

Предпроцедурный период включает в себя контакт пациентов с сотрудниками эндоскопии до приема седативных препаратов. В этот период пациент должен быть проинформирован о рисках и технических аспектах процедуры и подписать информированное согласие. Это период, когда команда, участвующая в процедуре, разрабатывает план приблизительной продолжительности соответствующей седации и принимает решение о профилактическом назначении антибиотиков [3]. Эндоскописты и другие сотрудники могут обсудить ожидаемые результаты процедуры и предположить продолжительность, которая может варьироваться в зависимости от сложности процедуры. Разработка плана и коммуникация в команде имеют важное значение для продолжительности седации и повышения качества.

Осознанное согласие

Информированное согласие должно быть сосредоточено на риске, связанном с эндоскопическими процедурами и ЭРХПГ в частности. Побочные эффекты должны быть четко объяснены и сосредоточены как минимум на шести возможных исходах: панкреатит, кровотечение, инфекция, сердечно-легочные осложнения, аллергическая реакция и перфорация. Пациенты должны быть проинформированы о побочных эффектах процедуры, о том, что процедура может оказаться неудачной и может потребоваться повторная процедура. Кроме того, следует прояснить, что побочные эффекты ЭРХПГ иногда отличаются и уникальны от таковых при стандартной эндоскопии.

Показания к проведению ЭРХПГ и документирование процедуры

Хорошо известно и устоявшееся правило, что ЭРХПГ должна выполняться по соответствующим показаниям [4-6]. Как правило, при выполнении ЭРХПГ без соответствующих показаний риск развития осложнений значительно выше. Соответствующее указание должно быть задокументировано для каждой процедуры, и если это необычное указание, причины этого должны быть четко указаны в документации. В таблице 1 приведен список подавляющего большинства допустимых показаний для ЭРХПГ.

Клинические условия, в которых ЭРХПГ, как правило, не показана, включают боль в животе без явных признаков заболевания поджелудочной железы, основанного на лабораторных или неинвазивных исследованиях визуализации [8,9]. Обычная ЭРХПГ перед холецистэктомией без обструкции желчевыводящих путей, холангита и камней в желчных протоках [7,10]. Облегчение злокачественной обструкции желчевыводящих путей у потенциально резектабельных пациентов. Несмотря на имеющиеся в литературе данные о том, что ЭРХПГ должна выполняться по соответствующим показаниям и не должна выполняться в вышеупомянутых сценариях, процедура по-прежнему широко используется в диагностических целях и для предоперационного дренирования желчевыводящих путей для декомпрессии желчевыводящих путей, особенно в развивающихся странах, где магнитно-резонансная холангиопанкреатография не является широко доступной и дорогостоящей [11]. Предоперационное дренирование оправдано только у пациентов, у которых операция будет отложена из-за неоадъювантной терапии или других методов предоперационной оценки. Эти случаи рассматриваются как надлежащая медицинская помощь.

Профилактические антибиотики

Антибиотики перед процедурой следует рассмотреть у пациентов с клиническими и/или лабораторными признаками холангита или у пациентов с подозрением на обструкцию желчевыводящих путей, у которых не удалось достичь полного дренирования желчного дерева [12].

Наконец, ЭРХПГ должен выполняться эндоскопистом, который полностью обучен и сертифицирован для этой процедуры. Объем случаев напрямую связан с показателем успеха и осложнениями и должен быть зарегистрирован. Исследование, проведенное в Австрии, показало, что у эндоскопистов с 50 ежегодными ЭРХПГ были более низкие показатели успеха и больше побочных эффектов во время ЭРХПГ, чем у врачей с большим объемом процедур [13]. Однако результаты Роттердамской формы самооценки ставят этот тезис под сомнение (см. Ниже). Кроме того, метаанализ, включающий девять исследований, 137 стажеров и 17100 процедур ЭРХПГ, показал огромную разницу в количестве процедур, необходимых для достижения компетентности. Эти цифры составляли от 79 до 400 процедур. Поэтому необходимо пересмотреть структуру программ повышения квалификации по эндоскопии, чтобы обеспечить достижение процедурной компетентности [14]. 
Внутрипроцедурный период

Внутрипроцедурный период ЭРХПГ - это период от начала процедуры и введения анестетика до извлечения эндоскопа. Этот период включает документирование технических аспектов процедуры и наблюдение за пациентом [3].

Седативное средство

Использование седативных средств для эндоскопии быстро развивается в последние годы, главным образом из-за необходимости повышения комфорта, удовлетворенности и безопасности пациентов при одновременном повышении эффективности эндоскопии. Общие правила при использовании седации при эндоскопии включают периодическую оценку и документирование жизненно важных показателей с частотой, которая зависит от вводимого лекарства, продолжительности процедуры и состояния пациента. Оборудование и медикаменты для экстренной реанимации должны быть в наличии. Требования к мониторингу пациента включают электронную оценку артериального давления, частоты сердечных сокращений, пульсоксиметрию и визуальную оценку дыхательной активности и сознания обученным специалистом.

Избранная группа пациентов может пройти эндоскопическую процедуру без седативных препаратов. В эту группу входят пожилые люди с сопутствующими заболеваниями и противопоказаниями для седации, не тревожные пациенты и без болей в животе в анамнезе. Обычно в этих случаях используются эндоскопы малого диаметра (назоэндоскоп или педиатрический гастроколоноскоп) для улучшения переносимости и комфорта пациента. Кроме того, использование инсуффляции воды и СО2 может улучшить общее состояние пациентов и уменьшить боль, вызванную процедурой $[15,16]$. Как правило, даже при неседированной эндоскопии должен быть доступен периферический венозный путь в случае возникновения сердечно-легочного незапланированного события и необходимости седации.

Важным фактором, способствующим седации, является введение новых агентов, таких как пропофол, который позволяет быстро вызвать глубокую седацию и восстановление после процедуры [17]. Недавний метаанализ, сравнивающий пропофол с другими традиционными седативными средствами, используемыми в эндоскопии, включая 22 рандомизированных контрольных исследования, в которых 1798 пациентов рассмотрели его безопасность и эффективность. Метаанализ показал, что использование пропофола связано с более короткими периодами восстановления и выписки, улучшением седативного эффекта и сотрудничеством с пациентами без увеличения сердечно-легочных осложнений. Не было никакой разницы в удовлетворенности пациентов по сравнению с традиционными седативными средствами.

ЭРХПГ - это сложная процедура, выполнение которой обычно занимает больше времени, чем обычная гастроскопия/колоноскопия. Недавнее исследование, в котором оценивались пациенты, проходящие ЭРХПГ под сознательной седацией, показало, что половина пациентов испытывала боль и дискомфорт во время процедуры и в период после процедуры [18]. Другое исследование показало, что частота отказов ЭРХПГ была двойной (14\% по сравнению $7 \%$ при использовании сознательной седации по сравнению с общей анестезией. Эта более высокая частота отказов была вызвана преждевременным прекращением лечения, вызванным неадекватной седативной терапией [19]. До сих пор продолжаются споры о наиболее подходящем седативном средстве для сложных эндоскопических процедур, таких как ЭРХПГ. Недавний кокрейновский обзор выявил четыре РКИ, в которых сравнивалась умеренная седативная терапия мидазоламом и меперидином с пропофолом. Не было никакой разницы в смертности, серьезных сердечно-легочных осложнениях или удовлетворенности пациентов между двумя методами седации. Единственным отличием было более быстрое и лучшее восстановление в группе пропофола [20].

Седативная терапия при ЭРХПГ различается в разных центрах и у разных анестезиологов. Варианты на выбор - глубокая седация пропофолом и общая анестезия. Решение принимается на основе процедурных характеристик и характеристик пациента. Некоторые центры предпочитают интубировать только сложные случаи, для завершения которых, как ожидается, потребуется больше времени. Другие предпочитают выполнять всю ЭРХПГ под общим наркозом, и для этого есть веская причина, хотя такой подход не является ни разумным, ни желательным. Главным аргументом в их защиту является то, что анестезиолог не знаком с обстановкой в кабинете эндоскопии и, возможно, не имеет всего оборудования, лекарств и вспомогательного персонала, с которыми они привыкли работать в операционной [21]. Кроме того, существуют более ранние исследования, которые указывают на повышенный риск осложнений и смерти, если анестезия проводится вне операционной в так называемых отдаленных местах. Эти исследования стали ориентиром для установления правил седации сегодня.

Сложность ЭРХПГ является переменной, со шкалой сложности от 1-4 классов, предложенной Комитетом по качеству ASGE [22]. Классы 1 и 2 считаются менее сложными и должны быть пройдены за относительно более короткое время по сравнению с более сложными классами 3 и 4. Общее мнение между центрами заключается в том, что, если характеристики пациента не диктуют общую анестезию, обычно ЭРХПГ 1 и 2 степени и даже многие процедуры 3 степени могут проводиться с глубокой седацией пропофолом без эндотрахеальной интубации. Ожидаемые сложные процедуры, для завершения которых потребуется больше времени, должны быть интубированы в интересах безопасности и переносимости пациента. Таким образом, для 
повышения качества ЭРХПГ следует четко сформулировать и принять в качестве обычной практики, чтобы эндоскописты обсуждали каждую процедуру ЭРХПГ с анестезиологами, чтобы помочь в принятии решений. Важно сообщить об ожидаемой сложности процедуры и предполагаемом времени ее выполнения, а также о любых известных проблемах с желудочно-кишечным трактом, которые могут повлиять на принятие решения, таких как повышенный риск аспирации, связанный с известным гастропарезом или обструкцией выходного отверстия желудка.

Документация по техническим аспектам должна включать достижение глубокой канюляции желаемого канала, документирование установки стента и извлечения камня.

Скорость канюлирования

Чтобы успешно выполнить ЭРХПГ, эндоскопист должен сначала провести канюлю в желаемый проток, что считается одним из наиболее сложных аспектов всей процедуры. Вот почему канюляция и неудачные попытки канюлирования должны регистрироваться во всех случаях. Конкретные критерии исключения неудачной канюляции включают неадекватную седативную терапию, сохраненное содержимое желудка, предшествующие операции на брюшной полости, такие как панкреатодуоденэктомия, гастроеюностомия и гепатоеюностомия, а также непроходимость антрального отдела и проксимального отдела двенадцатиперстной кишки. Успешная канюляция должна быть измерена на неповрежденном сосочке и, по сути, означает глубокую канюляцию желаемого протока с возможностью введения контраста и облегчения введения различных инструментов.

Недавний метаанализ с использованием модели случайных эффектов предполагает, что частота канюлирования на практике, даже в центрах третичной медицинской помощи, может составлять $90 \%$ (в среднем диапазоне $80 \%$ ) и предполагает значительную разницу в частоте канюлирования в развитых странах мира [23]. Некоторые исследовательские группы опубликовали данные об успешной канюляции в диапазоне 92-94\% [1517,24-26].

Основываясь на обзоре литературы, целевая группа ASGE /ACG предполагает, что врачам с неизменно субоптимальными показателями канюлирования (80\% успеха) следует рассмотреть возможность прохождения дальнейшего обучения или прекращения практики ЭРХПГ [1].

Добыча камня

Добыча камня также должна быть задокументирована. Протокол после ЭРХПГ должен включать данные о размере, количестве, местоположении и о том, достигнуто ли полное извлечение. Также должна быть включена информация о рентгеноскопической анатомии желчного дерева - наличие стриктур или аномалий. Согласно одному исследованию, экспертные центры эндоскопии могут добиться удаления камней независимо от их размера более чем у 90\% пациентов [27]. Конечно, это включает в себя использование дополнительных методов, таких как механическая, лазерная или электрогидравлическая литотрипсия, когда стандартные методы терпят неудачу. Другое исследование показывает, что компетентные эндоскописты ЭРХПГ могут очистить проток от камней общего желчного протока малого и среднего размера диаметром до 1 см в 90\% случаев с помощью сфинктеротомии и удаления камней баллоном или корзиной у пациентов с нормальной анатомией желчевыводящих путей [28]. Сложные крупные камни обычно устраняются с помощью баллонной сфинктеропластики. Основным преимуществом процедуры является снижение риска кровотечения и перфорации [29]. Однако риск развития панкреатита после ЭРХПГ (РЕР) выше, если пациентам проводится баллонная дилатация без ранее выполненной сфинктеротомии [29,30]. Наконец, ASGE утверждает, что успешное извлечение камней должно быть достигнуто более чем в $85 \%$ случаев.

Установка стента

Документация по установке стента должна быть включена в каждый протокол после ЭРХПГ. Документация должна включать информацию об успешном введении стента и достаточную информацию о показаниях, расположении стриктуры, размере и типе стента, а также о наличии послеоперационной анатомии. Это облегчит сравнение и последующие контрольные работы. Обзор литературы показывает, что компетентные эндоскописты ЭРХПГ должны быть в состоянии установить билиарный стент для облегчения нехилярной обструкции желчевыводящих путей более чем у 90\% пациентов [31,32].

Рентгеноскопия

Время рентгеноскопии и доза облучения должны быть измерены и задокументированы. Радиационное облучение должно быть задокументировано и снижено до минимально возможного уровня, хотя некоторые процедуры ЭРХПГ являются более сложными, чем другие, и требуют более длительного общего времени рентгеноскопии. Одно исследование показало, что опытные эндоскописты имеют значительно более короткое время облучения по сравнению с менее опытными [15]. Также следует отметить, что доза облучения варьируется в зависимости от габитуса тела пациента, использования медной фильтрации, расстояния пациента до источника излучения, увеличения, наклонного обзора и точечных изображений.

Период после процедуры

Период после процедуры - это время от удаления эндоскопа до последующего наблюдения. В период после процедуры эндоскопист должен подготовить полный отчет о процедуре, в котором описываются конкретные выполненные методы, используемые принадлежности и общий результат. Отчет должен включать 
документацию о неблагоприятных явлениях, если таковые имеются. После процедуры эндоскопист должен объяснить пациенту результат и дать соответствующие инструкции. Кроме того, общение с лечащим врачом и последующее наблюдение за патологией, мягко говоря, желательно.

Отчет ЭРХПГ

Отчет ЭРХПГ должен включать фото- или видеодокументацию процедуры, включая рентгеноскопические изображения, достижение канюлирования или невыполнение этого, четкое описание событий в последовательном порядке и общий результат. Следует также сообщить о непреднамеренной канюляции или инъекции с контрастированием протока поджелудочной железы. Четкое и надлежащее документирование результатов ЭРХПГ без сокрытия фактов помогает врачам, непосредственно занимающимся оказанием медицинской помощи пациентам, принимать соответствующие решения по ведению пациентов.

Неблагоприятные события

Признанные нежелательные явления, такие как кровотечение, аллергические реакции, сердечно-легочные осложнения (включая аспирацию), перфорация и РЕР, должны быть задокументированы.

Панкреатит после ЭРХПГ определяется как боль в животе после ЭРХПГ, сопровождающаяся уровнем амилазы и липазы в сыворотке крови, в три раза превышающим верхнюю границу нормы [33]. Обзор литературы показывает, что показатели РЕР находятся в диапазоне от 1\% до 7\%. Некоторые группы высокого риска исключены из этой статистики. Это пациенты с известной или подозреваемой дисфункцией сфинктера Одди и те, кто проходит эндотерапию поджелудочной железы, которым может потребоваться специальная профилактика РЕР, включая установку панкреатического стента или профилактическое применение нестероидных противовоспалительных препаратов [8,34-37].

Частота перфорации, возникающей во время ЭРХПГ, составляет от 0,1\% до 0,6\% [34]. Перфорация может быть результатом механического разрыва пищевода, желудка или двенадцатиперстной кишки в результате прохождения инструмента, сфинктеротомии или прохождения направляющих проводов. Перфорация может быть внутрибрюшной или забрюшинной. Хорошо установленные факторы риска перфорации во время ЭРХПГ включают анатомию Биллрота II или Ру-ан-У, предполагаемую дисфункцию сфинктера Одди, интрамуральную инъекцию контрастного вещества, сфинктеротомию, расширение желчных путей и длительные процедуры [38]. У пациентов, проходящих ЭРХПГ с нормальной анатомией, ожидаемая частота перфорации составляет менее $1 \%$.

Кровотечение, связанное с ЭРХПГ, в большинстве случаев возникает из-за применения электрокоагуляции и сфинктеротомии. Частота клинически значимых кровотечений, связанных с ЭРХПГ, составляет примерно 1\%, причем в большинстве случаев наблюдается легкое внутрипросветное кровотечение. Кровотечение обычно делится на немедленное кровотечение во время процедуры или отсроченное кровотечение, которое может произойти через 10 дней после процедуры. Отсроченное кровотечение распознается по клиническим признакам, таким как мелена, при снижении уровня гемоглобина или необходимости переливания крови в течение 10 дней после ЭРХПГ. Ожидаемая частота серьезных кровотечений после сфинктеротомии может достигать 2\%. Факторы риска кровотечения после сфинктеротомии включают коагулопатию, холангит, антикоагулянтную терапию в течение 3 дней после процедуры и низкий объем обращений к эндоскописту (<1 в неделю). Однако риск кровотечения после процедуры выше, когда выполняются другие терапевтические маневры, такие как ампулэктомия и трансмуральное дренирование псевдокист .

Отсроченные побочные эффекты должны быть задокументированы путем обращения к пациентам в течение 14 дней после процедуры, если это возможно.

Сеть качества ЭРХПГ

Пилотный проект под названием сеть качества ЭРХПГ был создан в 2013 году в Соединенных Штатах в попытке улучшить оценку качества ЭРХПГ. Идея состоит в том, чтобы собрать и сравнить данные о практике и качестве ЭРХПГ отдельными эндоскопистами. Затем эти данные загружаются на защищенный веб-сайт, автоматически анализируются, и результаты немедленно публикуются. Точки данных включают вышеупомянутые критерии, такие как показания, степень сложности, седативное действие, время рентгеноскопии, показатели успешности глубокой канюляции, стентирования и т.д. Дополнительным преимуществом является то, что каждый эндоскопист может просмотреть сводку своей собственной работы и сравнить ее с другими участниками. За первые 6 месяцев исследования 24 эндоскописта Соединенных Штатов провели 2300 процедур ЭРХПГ, и результаты были аналогичны рекомендованным стандартам ASGE. Частота глубокой канюляции желчевыводящих путей составила в среднем 95\%, показатели успеха при желчных камнях менее 10 мм составили 97\%, снизившись до 89\% при камнях выше 10 мм. и общая частота зарегистрированных осложнений составила $3 \%$ легкой степени и $1 \%$ средней степени тяжести .

Программа самооценки

Недавно исследовательская группа из медицинского центра Эразмус в Роттердаме, Нидерланды, предложила программу самооценки для лучшего измерения качества эндоскопии. Роттердамская форма оценки ЭРХПГ (RAF-E) была основана на форме, заполненной каждым эндоскопистом, участвовавшим в исследовании. Форма была заполнена по завершении процедуры и включала объективный и субъективный критерий. Несмотря 
на некоторые недостатки, которые имело исследование, например, оно проводилось в одном высшем учебном заведении, а не в масштабах всей страны, и влияние стажеров не было установлено, оно дало очень интересные результаты. Несмотря на относительно небольшой объем включенных процедур ЭРХПГ - 1651, был сделан вывод об отсутствии корреляции между объемом и производительностью практикующих эндоскопистов. Тот, у кого была самая низкая громкость, не был худшим исполнителем, и наоборот. В целом это доказало, что простая форма самооценки может быть ценным методом для получения информации о результатах ЭРХПГ .

Выводы

ЭРХПГ является одной из наиболее технически сложных процедур в гастроэнтерологии, с обязательной кривой обучения для освоения и определенным уровнем процедур для поддержания мастерства. Клинические преимущества ЭРХПГ очень прочно доказаны, но в последние годы растет озабоченность по поводу качества процедур и того, как именно его следует измерять. Различные эндоскопические организации опубликовали свои руководящие принципы и предложения по мерам качества, но их несколько сложно применять в повседневной практике. Возможно, единая международная программа оценки качества, содержащая вышеупомянутые параметры, является способом достижения оценки качества ЭРХПГ. Таким образом, недавно созданная сеть качества ЭРХПГ и программа самооценки могут дать наиболее точное представление о проблеме, хотя необходимо провести дальнейшие исследования.

\section{Исползованная литература}

1. М.Т. Баракат и др. Связанные с трансплантацией печени анастомотические стриктуры желчных путей: новый, быстрый, безопасный, щадящий радиацию и экономически эффективный подход к лечению. Гастроинтест Эндоскоп (2018).

2. Дж.Х. Табибиан и др. Эндоскопическое лечение анастомотических желчных стриктур послеортотопической трансплантации печени с максимальной терапией стентом (с видео). Гастроинтест Эндоскоп (2010).

3. Г. Морелли и др. Быстрое последовательное эндоскопическое лечение посттрансплантационных анастомотических желчных стриктур. Гастроинтест Эндоскоп (2008).

4. Дж.П. Виолончель и др.Долгосрочное наблюдение за эндоскопической ретроградной холангиопанкреатографической сфинктеротомией у пациентов с папиллярным стенозом синдрома приобретенного иммунодефицита. Am J Med (1995).

5. Дж.Л. Буксбаум и др. Руководство ASGE по роли эндоскопии в оценке и лечении холедохолитиаза. Гастроинтест Эндоскоп (2019).

6. М.А. Хашаб и др. Антибиотикопрофилактика при эндоскопии желудочно-кишечного тракта. Гастроинтест Эндоскоп (2015).

7. У. Наваниэтан и др. Побочные эффекты, связанные с ЭРХПГ, у пациентов с первичным склерозирующим холангитом. Гастроинтест Эндоскоп (2015).

8. С.Ү. Понсиоен и др. Нет преимущества стентов по сравнению с баллонной дилатацией при доминирующих стриктурах у пациентов с первичным склерозирующим холангитом. Гастроэнтерология (2018).

9. М. Кайя и др. Баллонная дилатация по сравнению со стентированием доминирующих стриктур при первичном склерозирующем холангите. Аm J Гастроэнтерол (2001).

10. А.В. ван Миллиген де Вит и др. Отсутствие осложнений после кратковременной терапии стентом при стриктурах внепеченочных желчных протоков при первичном склерозирующем холангите. Гастроинтест Эндоскоп (1997).

11. С.Л.Т. Теджасви и др. Холангиоскопия при первичном склерозирующем холангите: серия случаев доброкачественных находок. Видео GIE (2021).

12. Д.Н. Готтхардт и др. Эндоскопическое расширение доминирующих стенозов при первичном склерозирующем холангите: результат после длительного лечения. Гастроинтест Эндоскоп (2010).

13. Н. Каззагон и др. Прогностические критерии ответа на эндоскопическое лечение тяжелых стриктур при первичном склерозирующем холангите. Клинический Гепатол Гастроэнтерол (2019).

14. С. Хан и др. Конфокальная лазерная эндомикроскопия на основе зонда в оценке доминирующих стриктур у пациентов с первичным склерозирующим холангитом: результаты многоцентрового проспективного исследования в США. Гастроинтест Эндоскоп (2021).

15. Н. Азим и др. Холангиоскопия с узкополосной визуализацией у пациентов с первичным склерозирующим холангитом, проходящих ЭРХПГ. Гастроинтест Эндоскоп (2014).

16. У. Наваниэтан и др. Гибридизация флуоресценции in situ для диагностики холангиокарциномы при первичном склерозирующем холангите: систематический обзор и мета-анализ. Гастроинтест Эндоскоп (2014).

17. К.М. Боберг и др. Диагностическая польза цитологии желчных путей при холангиокарциноме при первичном склерозирующем холангите. Ј Гепатол (2006). 
18. Г. Трикуданатан и др. Диагностический результат чистки желчных протоков при холангиокарциноме при первичном склерозирующем холангите: систематический обзор и мета-анализ. Гастроинтест Эндоскоп (2014).

А. Стил и др. Развитие стенозов доминирующих желчных протоков у пациентов с первичным склерозирующим холангитом, получавших урсодезоксихолевую кислоту: результат после эндоскопического лечения. Ј Гепатол (2002)

19. А.А. Госсард и др. Холестатическое заболевание печени. Медицинский центр Северной Америки (2014).

20. С. Влэдуц и др. Обзор первичного склерозирующего холангита. J Clin Med (2020).

21. Роль эндоскопии при первичном склерозирующем холангите: клиническое руководство Европейского общества эндоскопии желудочно-кишечного тракта (ESGE) и Европейской ассоциации по изучению печени (EASL). J Гепатол (2017)

22. С. Вебер и др. Магнитно-резонансная холангиопанкреатография в диагностике первичного склерозирующего холангита. Эндоскопия (2008).

23. П. Хаммель и др. Регрессия фиброза печени после дренирования желчевыводящих путей у пациентов с хроническим панкреатитом и стенозом общего желчного протока. N Английский J Мед (2001).

24. М.Х. Чепмен и др. Холангиокарцинома и доминирующие стриктуры у пациентов с первичным склерозирующим холангитом: 25-летний опыт работы в одном центре. Eur J Гастроэнтерол Гепатол (2012).

25. П. Чарачароенвиттхая и др. Полезность сывороточных онкомаркеров, визуализации и цитологии желчных путей для выявления холангиокарциномы при первичном склерозирующем холангите. Гепатология (2008).

26. Б.Л. Витт и др. Идентификация факторов, предикторных злокачественных новообразований у пациентов с атипичными результатами чистки желчевыводящих путей, полученными с помощью ЭРХПГ. Диагноз Цитопатол (2013).

27. E.G. Барр Фритчер и др. Пациенты с первичным склерозирующим холангитом с результатами последовательной гибридизации флуоресценции полисомии in situ подвергаются повышенному риску холангиокарциномы. Аm J Гастроэнтерол (2011).

28. С.Я. Бангарулингам и др. Отдаленные результаты положительных тестов гибридизации флуоресценции in situ при первичном склерозирующем холангите. Гепатология (2010).

29. А.Д. Сингхи и др.Интеграция секвенирования следующего поколения с полученными при эндоскопической ретроградной холангиопанкреатографии (ЭРХПГ) образцами желчных путей улучшает выявление и лечение пациентов со злокачественными стриктурами желчных протоков. Кишечник (2020).

30. Т.А. Францини и др. Достижения в области терапевтической холангиоскопии. Лечение Гастроэнтерола. (2016)

31. Б. Ньеи и др. Систематический обзор с метаанализом: методы диагностики холангиокарциномы при первичном склерозирующем холангите на основе эндоскопической ретроградной холангиопанкреатографии. Фармакология пищевых продуктов (2016).

32. У. Арнело и др. Перспективная оценка клинической полезности однооперационной пероральной холангиоскопии у пациентов с эндоскопией первичного склерозирующего холангита (2015).

33. Ю. Ишида и др. Виды пероральной холангиоскопии: как выбрать наиболее подходящий тип холангиоскопии. Варианты лечения Curr Гастроэнтеролом (2016).

А. Маджид и др. Оптимизация выявления дисплазии желчевыводящих путей при первичном склерозирующем холангите перед трансплантацией печени. Скандальный гастроэнтерол (2018)

34. Ф. Прат и др. Влияние пероральной холангиоскопии на лечение неопределенных заболеваний желчевыводящих путей: многоцентровое проспективное исследование. Передовой гастроэнтерол (2019).

35. К.Д. Линдор и др. Клиническое руководство ACG: первичный склерозирующий холангит. Am J Гастроэнтерол (2015).

36. М.X. Чепмен и др. Британское общество гастроэнтерологов и руководство UK-PSC по диагностике и лечению первичного склерозирующего холангита. Кишка (2019).

37. С.Я. Бангарулингам и др. Отдаленные результаты положительных тестов гибридизации флуоресценции in situ при первичном склерозирующем холангите. Гепатология (2010).

38. А.Д. Сингхи и др. Интеграция секвенирования следующего поколения с полученными при эндоскопической ретроградной холангиопанкреатографии (ЭРХПГ) образцами желчных путей улучшает выявление и лечение пациентов со злокачественными стриктурами желчных протоков. Кишка (2019). 